\title{
Phenomenon-based evaluation of relative biological effectiveness of ion beams by means of the multiscale approach
}

\author{
Alexey Verkhovtsev ${ }^{1,2,3^{*}}$ (D), Eugene Surdutovich ${ }^{4}$ and Andrey V. Solov'yov ${ }^{2,3}$
}

\author{
*Correspondence: \\ a.verkhovtsev@dkfz-heidelberg. \\ de \\ ${ }^{1}$ Division of Medical Physics \\ in Radiation Oncology, German \\ Cancer Research Center (DKFZ), \\ Im Neuenheimer Feld 280, \\ 69120 Heidelberg, Germany \\ Full list of author information \\ is available at the end of the \\ article
}

\begin{abstract}
Background: Relative biological effectiveness (RBE) is a key quantity for the description of radiobiological effects induced by charged-particle irradiation in the context of ion-beam cancer therapy. Since RBE is a complex function that depends on different physical, chemical, and biological parameters, a fundamental understanding of radiobiological effects becomes increasingly important for clinical applications. The phenomenon-based multiscale approach to the physics of radiation damage with ions (MSA) provides a tool for a molecular-level understanding of physical and chemical mechanisms of radiation biodamage and allows for quantification of macroscopic biological effects caused by ion irradiation. This study reports the first application of the MSA for the analysis of RBE of ion beams.
\end{abstract}

Results: As a case study, we quantify the response of human and rodent normal cells to carbon-ion irradiation at different values of linear energy transfer (LET). Clonogenic cell survival as a function of dose, RBE at a 10\% survival level as well as other radiobiological parameters, such as the cross section of cell inactivation, are analyzed and compared with experimental data. The MSA is used also to evaluate RBE at high values of LET where RBE decreases due to the "overkill" effect. In this regime, the dose needed to achieve a given biological effect is deposited by only a few ions and the RBE becomes inversely proportional to LET.

Conclusions: Good agreement with a large set of experimental data on clonogenic cell survival, inactivation cross section, and RBE emphasizes the predictive power of the MSA. In the high-LET regime, the fact that a given number of ions may produce more damage than needed for a given biological effect leads to a significant (up to 20\%) variation in RBE. Consideration of this effect in the analysis of experimental data on irradiation with high-LET ions may lead to re-evaluation of RBE in this regime.

Keywords: Ion-beam cancer therapy, Multiscale approach, Relative biological effectiveness, Cell survival, Overkill effect

\section{Background}

Ion-beam cancer therapy (IBCT), also known as hadron therapy, is an emerging, rapidly developing treatment technique (Schardt et al. 2010; Jäkel et al. 2008; Loeffler and Durante 2013; Mohamad et al. 2017). IBCT provides advances in cancer treatment due to the possibility of high dose localization in the tumor region. This allows maximizing cell killing within the tumor whilst simultaneously minimizing the radiation

(c) The Author(s) 2019. This article is distributed under the terms of the Creative Commons Attribution 4.0 International License (http://creativecommons.org/licenses/by/4.0/), which permits unrestricted use, distribution, and reproduction in any medium, provided you give appropriate credit to the original author(s) and the source, provide a link to the Creative Commons license, and indicate if changes were made. The Creative Commons Public Domain Dedication waiver (http://creativecommons.org/publi cdomain/zero/1.0/) applies to the data made available in this article, unless otherwise stated. 
damage to surrounding healthy tissue. IBCT is therefore appealing for the treatment of tumors that are radioresistant against conventional photon treatments or anatomically located next to critical and radiosensitive structures. In particular, it has been applied in clinic to treat different head and neck cancers (e.g., base of skull tumors, malignant salivary gland tumors), pancreatic and prostate cancers [see the review (Jensen et al. 2011) and references therein]. As of March 2019, there are 92 hadron therapy facilities in operation in 20 countries around the world, including 12 centers where carbon ions are used (Particle Therapy Co-Operative Group, https://www. ptcog.ch/index.php/facilities-in-operation). Other 45 facilities are currently under construction and should start operation within the next several years (Particle Therapy Co-Operative Group https://www.ptcog.ch/index.php/facilities-under-constructi on).

The advantages of IBCT over conventional radiotherapy with photons stem from the fundamental difference between the energy deposition profiles for heavy charged projectiles and photons (Schardt et al. 2010; Surdutovich and Solov'yov 2014). The energy deposited by the latter rises at shallow penetration depths and then decreases exponentially as photons are absorbed by tissue. On the contrary, the profile for ions is characterized by a plateau region followed by the Bragg peak-a sharp maximum in the depth-dose curve close to the end of ions' trajectories (Hall and Giaccia 2018). The position of the Bragg peak can be precisely adjusted to the desired depth by changing the kinetic energy of incident ions. Typical depths for carbon ions range from about 2.5 to $28 \mathrm{~cm}$ corresponding to the initial energy of ions ranging from 100 to $430 \mathrm{MeV} / \mathrm{u}$ (Schardt et al. 2010; Surdutovich and Solov'yov 2014).

There is an abundance of experimental evidence that irradiation with energetic ion beams results in enhanced cell killing as compared to photon irradiation at the same dose. The concept of relative biological effectiveness (RBE) has been introduced to account for this effect (IAEA 2008). RBE is defined as the ratio of a dose of photons to a dose of ions (or, in general, of any other radiation modality) leading to the same biological effect,

$$
\mathrm{RBE}=\frac{d_{\mathrm{ph}}}{d_{\text {ion }}} .
$$

This expression allows to calculate, for a given ion dose absorbed, the isoeffective photon dose and thus estimate the biological effect of ion irradiation on the basis of the wellknown response to a reference photon beam (Karger and Peschke 2018). Despite being a simple concept, RBE depends on many physical [e.g., radiation type, energy, linear energy transfer (LET), radiation dose, dose rate, fractionation scheme], chemical (e.g., oxygen concentration in the target), and biological (biological endpoint, intrinsic radiosensitivity of a given cell line, cell cycle phase, proliferation rate, etc.) parameters (Surdutovich and Solov'yov 2014; Karger and Peschke 2018; Paganetti 2014).

RBE can be determined for different biological endpoints. The effects of ion beams have been studied mostly in biological systems in vitro with clonogenic cell survival being a commonly used endpoint. In this case, irradiations with photons and ions are considered to be isoeffective if the dose-dependent survival fractions measured in the clonogenic assay are the same. 
Different radiobiological models have been developed to describe experimental outcomes and understand how physical parameters of irradiation impact the biological response of cells and tissues (Bodgi et al. 2016). The most widely known approaches are the Local Effect Model (LEM) (Schardt et al. 2010; Scholz et al. 1997; Elsässer et al. 2008; Friedrich et al. 2012) that is used for treatment planning in ion-beam centers in Europe, Microdosimetric Kinetic Model (MKM) (Hawkins 1996, 2003) as well as the modified MKM (MMKM) (Inaniwa et al. 2010; Kase et al. 2011) which is used clinically in Japan. The LEM describes biological effects of ion beams on the basis of amorphous track structure in combination with the known dose-response curves for photon radiation. The MKM and MMKM rely on microdosimetric concepts and on the estimation of the stochastic energy deposition into volumes of micrometer dimensions (Kelleler 1985). Several other approaches have been developed to explore radiation-induced DNA damage and repair and to relate these phenomena to cell inactivation. These approaches can be grouped into phenomenological models such as the Lethal-Potentially Lethal (Curtis 1986), Repair-Misrepair-Fixation (Carlson et al. 2008; Frese et al. 2012) or RepairableConditionally Repairable (Lind et al. 2003; Brahme and Lind 2010) models, and track structure based biophysical models (Cucinotta et al. 1999; Friedland et al. 2012, 2017) that provide a more detailed description of the passage of radiation through matter and its stochastic nature.

In the aforementioned approaches the radiobiological effect of ions is commonly quantified by means of an empirical linear-quadratic (LQ) model,

$$
-\ln \Pi=\alpha d+\beta d^{2},
$$

where $\Pi$ is a surviving fraction of cells exposed to a given dose of radiation $d$. The coefficients $\alpha$ and $\beta$, which characterize the response of biological systems to ion radiation, are usually derived using the photon dose-response curves. It should be noted that when tissue is exposed to $\mathrm{x}$-rays, the dose distribution in the plane perpendicular to the beam axis on the cellular $(10 \mu \mathrm{m})$ scale is uniform. Therefore, a model for determining the probability of cell survival can be built on a single physical quantity such as dose. With ions, the above picture changes since the radial distribution of dose varies on the nanometer scale and the dose on the cellular and sub-cellular scales becomes a probabilistic function of a number of ions traversing a given target (Surdutovich and Solov'yov 2014). In this case, physical parameters, such as number density of reacting species or their fluence, describe radiation damage in a more straightforward way.

The LEM and MMKM models are currently used in clinical practice for the dose optimization and treatment planning. However, recent studies observed a systematic discrepancy between the predictions of different versions of LEM (including the clinically used LEM I model) and in vivo experimental data (Saager et al. 2018). Therefore, despite a successful application of these models in clinic, there is a need for further improvement of the current therapy protocols and for the development of novel radiobiological models. Being based on an empirical Eq. (2), the existing models cannot answer many questions concerning the molecular-level mechanisms of radiation damage with ions. The understanding of radiation biodamage on a fundamental quantitative level may bring IBCT planning to a higher scientific level in order to design the new generation of radiotherapy protocols. 
The empirical level of the earlier concepts triggered formulation of the multiscale approach to the physics of radiation damage with ions (MSA) (Surdutovich and Solov'yov 2014; Solov'yov et al. 2009; Solov'yov 2017). It has been developed specifically for ions to construct an inclusive scenario of processes leading to radiation damage with the ultimate goal of its quantitative assessment. The key phenomena and processes addressed by the MSA are ion stopping in the medium, production of secondary electrons and free radicals as a result of ionization and excitation of the medium, transport of these species, the interaction of secondary particles with biomolecules, the analysis of induced biodamage, and the evaluation of the probabilities of subsequent cell survival. A unique feature of the MSA that is absent in other radiobiological models is the analysis of thermo-mechanical damage due to nanoscale shock waves that are induced by highLET ions traversing a biological medium (Surdutovich and Solov'yov 2010). A comprehensive description of different aspects of the MSA was given in earlier publications (Surdutovich and Solov'yov 2014; Solov'yov 2017). The predictability of cell survival by the MSA was tested on a variety of cell lines with different values of LET and oxygenation conditions (Verkhovtsev et al. 2016). Another recent achievement of the MSA is formulation of a recipe for solving a problem of variable cell survival probability along the spread-out Bragg peak (Surdutovich and Solov'yov 2017).

In this paper the MSA methodology is applied to evaluate RBE of ion beams. As a case study, we analyze the response of human and Chinese hamster normal tissue cell lines to single-fraction carbon-ion irradiation. We consider different values of LET in the range from several tens of $\mathrm{keV} / \mu \mathrm{m}$ up to about $100 \mathrm{keV} / \mu \mathrm{m}$, which corresponds to the LET range of interest for clinical applications of carbon ions. ${ }^{1}$ Clonogenic cell survival curves, RBE at different doses and survival levels as well as the cell inactivation cross section are analyzed and compared with experimental data for HE, NB1RGB, HFL-III, M/10, and V79 cell lines available in the literature and compiled in the Particle Irradiation Data Ensemble (PIDE) database (Friedrich et al. 2013b). A good agreement with experimental results illustrates the capability of the MSA to quantitatively describe RBE and other radiobiological parameters. Finally, the MSA is used to evaluate RBE at high values of LET (above $100 \mathrm{keV} / \mu \mathrm{m}$ ) where RBE for carbon ions is known to decrease due to the "overkill" effect (Linz 2012). The fact that a given number of high-LET ions may produce more damage than needed for a given biological effect leads to a significant variation of RBE. Normal cell lines are chosen as an illustrative case study because their proliferation is highly organized as compared to tumor cells. This allows us to test further the MSA methodology and justify the choice of its key parameters, e.g., the genome size which remains almost constant in normal cells but may vary greatly in different tumor cells (Kops et al. 2005).

\footnotetext{
1 The clinically relevant LET range in IBCT is several orders of magnitude larger than that in photon irradiation. In the latter case the LET varies from approx. $2 \mathrm{keV} / \mu \mathrm{m}$ for $200-\mathrm{kV}$ x-rays down to $0.2 \mathrm{keV} / \mu \mathrm{m}$ for $22-\mathrm{MV}$ x-rays (International Commission of Radiation Units and Measurements 1970).
} 


\section{The MSA methodology}

The assessment of RBE for ions within the MSA starts from the calculation of survival curves for a given type of cells irradiated with a given type of ions at given conditions. This requires establishing of the relation between the above-described physical effects and radiation damage. In regard to irradiation with ions, the key assumption adopted in the MSA, following Ward (1995), Amaldi and Kraft (2005) and Malyarchuk et al. (2009), is that the leading cause of cell inactivation is the complexity of nuclear DNA damage.

The criterion for lethality of damage suggested in Surdutovich and Solov'yov (2014) is based on the well-established hypothesis that among different DNA lesions caused by the interaction with secondary electrons and other reactive species (e.g., free radicals and solvated electrons) the multiple damaged sites with sufficient complexity may not be repaired (Ward 1995; Sage and Harrison 2011; Malyarchuk et al. 2009). In the formulated recipe for the assessment of biodamage, it was postulated that a complex lesion combined of a double-strand break (DSB) and at least two other simple lesions such as single-strand breaks (SSBs) within two DNA twists is lethal for a cell (Surdutovich and Solov'yov 2014). In our previous study (Verkhovtsev et al. 2016) this criterion was justified and applied successfully to a number of cell lines.

The multiple damage sites contain several lesions, each of which is caused by independent agents, such as secondary electrons, free radicals, or solvated electrons (Surdutovich et al. 2011). The MSA calculates the probability of such a site to be formed at a distance $r$ from an ion's path; then the space averaging is applied. Details of this methodology are given below.

\section{Transport of secondary particles}

Secondary electrons produced following the ion's passage propagate in the medium on the femtosecond time scale (Surdutovich and Solov'yov 2015). They react with DNA molecules producing lesions such as SSBs, DSBs, base damages, etc. The typical range for most of secondary electrons is within several nanometers from the ion's path and the diffusion mechanism describes their transport adequately. Less abundant $\delta$-electrons (which are kinematically allowed to form in the plateau region of a Bragg curve) may induce damage sites several hundreds of nanometers away from the ion's path.

Mechanisms of transport of reactive species depend on the ion's LET. If the LET is relatively small (corresponding to the entrance channel or the plateau region of a Bragg curve), free radicals and solvated electrons are formed in rather small numbers on a picosecond time scale and diffuse away from the ion's path reaching their targets on the way. The lifetime of these species is limited by their interactions with each other and with other components of the medium and, provided their number densities are small enough, can be rather long, up to $10^{-4} \mathrm{~s}$ (von Sonntag 1987; Alpen 1997). Such long times may largely increase the distances the reactive species could propagate. However, as the reactive species diffuse out, their number density decreases and may fall below the minimum density required for the formation of a lethal lesion. Such a condition of the required minimum number density becomes the limiting factor for the effective range of reactive species propagation. 
The condition of the required minimum number density is introduced as a logical consequence of the introduction of the criterion for lesion lethality and the understanding that the formation of a complex lesion requires a certain number of agents. The introduction of this condition is natural in the framework of the MSA. As the criterion itself is understood better the condition can be correspondingly modified. This is another reason why the MSA raised the interest to physical mechanisms of formation of lesions such as DSBs trying to understand how many secondary electrons or reactive species are required for their production.

At higher values of LET (corresponding to that in the Bragg peak region), the reactive species are produced in larger numbers. The high reaction rates for interactions of reactive species may lead to their recombination and not allow them to leave a few-nm ion track. A different physics, namely the predicted ion-induced shock waves (Surdutovich and Solov'yov 2010), steps in the scenario of radiation damage. The collective radial flow induced by these waves carries the reactive species, hence reducing their number densities and saving them from recombination (Surdutovich and Solov'yov 2015). This process happens on a picosecond time scale, and the radial range to which the reactive species can propagate is determined by the strength of the shock wave. This effect is complex and can be studied by means of advanced molecular dynamics simulations (de Vera et al. 2018; Surdutovich et al. 2013; Fraile et al. 2019). The most up-to-date review of the effects induced by shock waves in relation to IBCT can be found in the paper by de Vera et al. (2019) in the same topical series.

Analytical considerations show that the effective range of reactive species propagation by the shock wave-induced collective flow is linear in the first order with respect to LET (Surdutovich et al. 2017). Indeed, as it was shown (Surdutovich and Solov'yov 2010) the pressure on the front of the shock wave is given by

$$
P(r)=\frac{1}{\gamma+1} \frac{\beta^{4}}{2} \frac{S_{\mathrm{e}}}{r^{2}},
$$

where $S_{\mathrm{e}}$ is the LET, $\gamma=C_{P} / C_{V} \approx 1.2$ is the heat capacity ratio for water molecules, $\beta=0.86$ is a dimensionless constant, and $r(t) \propto \sqrt{t}$ is the radius of the wave front. As the shock wave propagates in the radial direction away from the ion's path, it causes a rarefaction in its wake and a cylindrical cavity of the radius $r_{\text {in }}<r$ is formed. The radius of the wave front increases as the pressure drops; this happens until the force inside the cavity (due to surface tension pressure $\kappa / r_{\text {in }}$ with $\kappa$ being the coefficient of surface tension) equilibrates the tearing force (Surdutovich et al. 2017). The condition for saturation of the radial propagation of the shock wave-induced collective flow can be estimated by equating the pressure force acting on a fragment of the wave front and the force due to surface tension on the inner surface (Surdutovich et al. 2017),

$$
\frac{1}{\gamma+1} \frac{\beta^{4}}{2} \frac{S_{\mathrm{e}}}{r^{2}} 2 \pi r l=\frac{\kappa}{r} 2 \pi r l,
$$

where $r$ is considered to be the same on the left- and right-hand sides since the thickness of the wave front is much smaller than $r$. The hydrodynamic phase, roughly described by this equation, ends when the pressure becomes uniform again. As a result of this phase, the reactive species are expected to be uniformly distributed within the range $R$. After 
the hydrodynamic equilibrium is achieved the reactive species propagate further due to the diffusion mechanism, but this stage is only of interest to us if the hydrodynamic range is smaller than that given by the required minimum number density. The linear dependence of $R$ on LET follows from Eq. (4), however the numerical value of $R$ from that equation depends on the choice of $\kappa$, which is an uncertain quantity at the medium conditions arising in the shock wave. Comparison of this analysis with the molecular dynamics simulations (de Vera et al. 2016, 2018) show that shock waves decay on much shorter distances than it follows from Eq. (4) evaluated at ambient conditions. The range of propagation of reactive species by the shock wave and its dependence on LET are currently under more thorough investigation using the molecular dynamics simulations with reactive force fields (Sushko et al. 2016).

The secondary electron contribution to the scenario of DNA damage has been understood better than that of reactive species. A part of this understanding is that the damage is caused not by the number density of electrons but rather by the number of hits of a particular molecular target. Then, the number of electrons incident on a typical target (i.e., the total average fluence), $F_{\mathrm{e}}(r)$, multiplied by an average probability of producing a simple lesion (like a SSB) per hit, $\Gamma_{\mathrm{e}}$, gives the total average number of simple lesions produced at a distance $r$ from the path, $\mathcal{N}_{\mathrm{e}}(r)$ :

$$
\mathcal{N}_{\mathrm{e}}(r)=\Gamma_{\mathrm{e}} F_{\mathrm{e}}(r)=\Gamma_{\mathrm{e}} \int_{0}^{t_{1}} \Phi_{\mathrm{e}}(r, t) \mathrm{d} t .
$$

The number of secondary electrons incident on a target is calculated as an integral of the flux of secondary electrons through the target, $\Phi_{\mathrm{e}}(r, t)$, over time. This integral is taken from zero to the time $t_{1}$ on a femtosecond scale until when the electrons can be treated as ballistic particles. At the larger time scales, remaining electrons become solvated and are treated together with other reactive species created in the medium. The flux $\Phi_{\mathrm{e}}(r, t)$ is obtained by solving a three-dimensional diffusion equation (Surdutovich and Solov'yov 2014, 2015). Explicit analytical expressions for $\Phi_{\mathrm{e}}(r, t)$ and $F_{\mathrm{e}}(r)$ can be found in Surdutovich and Solov'yov (2014).

Even though the transport of reactive species is less understood at the moment, it is possible to cast it in the same form as that of secondary electrons. Then the complete picture looks as

$$
\begin{aligned}
\mathcal{N}(r) & =\mathcal{N}_{\mathrm{e}}(r)+\mathcal{N}_{\mathrm{r}}(r)=\Gamma_{\mathrm{e}} F_{\mathrm{e}}(r)+\Gamma_{\mathrm{r}} F_{\mathrm{r}}(r) \\
& =\Gamma_{\mathrm{e}} \int_{0}^{t_{1}} \Phi_{\mathrm{e}}(r, t) \mathrm{d} t+\Gamma_{\mathrm{r}} \int_{0}^{t_{2}} \Phi_{\mathrm{r}}(r, t) \mathrm{d} t,
\end{aligned}
$$

where quantities with index ' $r$ ' represent similar quantities for reactive species. The time limit $t_{2}$ depends on the physics involved in the transport of reactive species such as the shock wave-induced collective flow followed by hydrodynamic relaxation and diffusion. Thus, $t_{2}$ can be on the picosecond or even the nanosecond scale depending on the LET. In our approach we choose $t_{2}$ on the picosecond scale according to the aforementioned criterion of the formation of lethal lesions in the vicinity of the track due to the creation of sufficiently high density of reactive species. 
If the transport of reactive species were understood better, we would not have to discuss the detail of LET-dependence of quantities in Eq. (6) as the corresponding integrands would naturally decrease with time and the distance. However, since there is no sufficient understanding of this transport at the moment, we assume a linear dependence of the range of propagation of reactive species on LET following from Eq. (4), and take up a conservative estimate of $R \approx 10 \mathrm{~nm}$ for carbon ions at their Bragg peak (Surdutovich and Solov'yov 2014). Within this range, the density of reactive species is high enough to ensure the production of lethal DNA lesions as discussed above. Furthermore, following Surdutovich and Solov'yov (2014) and Verkhovtsev et al. (2016), the average number of lesions due to reactive species at a distance $r$ from the path is taken as

$$
\mathcal{N}_{\mathrm{r}}(r)=\mathcal{N}_{\mathrm{r}} \theta\left(R\left(S_{\mathrm{e}}\right)-r\right)
$$

where $\theta$ is the Heaviside function and $R=10 \mathrm{~nm} \times S_{\mathrm{e}} / S_{\mathrm{e}, \mathrm{CBP}}$ with $S_{\mathrm{e}, \mathrm{CBP}}$ being the LET of carbon ions at their Bragg peak. The value of $\mathcal{N}_{\mathrm{r}}$ was estimated as 0.08 from the comparison of the experimental results (Dang et al. 2011) for plasmid DNA dissolved in pure water and in a scavenger-rich solution. Further work is however needed to obtain more detailed dependencies of $N_{\mathrm{r}}$ and $R$ on LET.

\section{Evaluation of the number of lethal lesions and cell survival probability}

After $\mathcal{N}(r)$ is obtained, the probability of production of a lethal lesion at a distance $r$ from the path, $\mathcal{P}_{1}(r)$, can be calculated according to the criterion of lethality determined in Surdutovich and Solov'yov (2014) and Verkhovtsev et al. (2016),

$$
\mathcal{P}_{1}(r)=\lambda \sum_{\nu=3}^{\infty} \frac{[\mathcal{N}(r)]^{\nu}}{\nu !} \exp [-\mathcal{N}(r)]
$$

where $v$ is the number of simple lesions in a clustered damage and $\mathcal{N}(r)$ was defined in Eq. (6). The sum starts with $v=3$, which makes the minimum order of lesion complexity at a given site equal to three. The factor $\lambda$ is the probability that one of the simple lesions is converted to a DSB. This implies that in the current model the DSBs occur via SSB conversion but other mechanisms can also be taken into account (Surdutovich and Solov'yov 2012). The introduction of $\lambda$ relies on experimental findings (Huels et al. 2003; Sanche 2005) that the DSBs caused by electrons with energies higher than about $5 \mathrm{eV}$ happen in one hit. In this case, the subsequent break in the second strand of the DNA is due to the action of debris generated by the first SSB. In the cited works it was shown that if a single electron causes a SSB, the same electron causes a DSB with a probability of about $0.1-0.2$ of that to create a SSB. The value $\lambda=0.15$ was suggested and justified earlier (Surdutovich and Solov'yov 2014; Verkhovtsev et al. 2016) and has been utilized in the analysis presented below.

Equation (8) represents the radial distribution of lethal lesions. Integration of $\mathcal{P}_{1}(r)$ over the area perpendicular to the ion's path gives the number of lethal lesions per unit length of the ion's trajectory,

$$
\frac{\mathrm{d} N_{\mathrm{l}}}{\mathrm{d} x}=n_{\mathrm{s}} \int_{0}^{R} \mathcal{P}_{\mathrm{l}}(r) 2 \pi r \mathrm{~d} r=n_{\mathrm{s}} \sigma_{\mathrm{l}}\left(S_{\mathrm{e}}\right) .
$$


Here, $n_{\mathrm{s}}$ is the number density of chromatin which is proportional to the ratio of DNA base pairs accommodated in the cell nucleus to the nuclear volume, $n_{\mathrm{s}} \sim N_{\mathrm{bp}} / V_{\mathrm{n}}$ (Verkhovtsev et al. 2016). The upper integration limit in Eq. (9) corresponds to the range $R$ in the Heaviside function (7). This is a combination of ranges of transport by the collective flow, hydrodynamic relaxation, and the diffusion of radicals. As described above, the limiting factor is the minimal number density of reactive species at which the formation of lethal lesions is still possible. Processes that accomplish the transport depend on the LET and the shock wave-induced collective flow plays an important role by saving the reactive species from recombination (de Vera et al. 2018). Function $\sigma_{\mathrm{l}}\left(S_{\mathrm{e}}\right)$ is the cross section of production of a lethal damage in the cell nucleus. It depends on LET and also on environmental conditions of the target (e.g., on the concentration of oxygen). The dependence of $\sigma_{1}$ on $S_{\mathrm{e}}$ comes from the number of reactive species hitting the DNA (which is proportional to LET) and from the range of their propagation [which is nearly proportional to LET as follows from Eq. (4)]. Therefore, in the first approximation, one may write

$$
\sigma\left(S_{\mathrm{e}}\right)=\xi S_{\mathrm{e}}^{2}
$$

where $\xi=5.8 \times 10^{-6} \mathrm{~nm}^{4} / \mathrm{eV}^{2}$ is a coefficient. It was obtained by calculating $\sigma_{\mathrm{l}}\left(S_{\mathrm{e}}\right)$ using Eq. (9) and fitting the resulting dependence with a quadratic function of LET. Further details of calculation of parameters entering Eqs. (6)-(10) can be found in Surdutovich and Solov'yov (2014) and Solov'yov (2017).

The effect of each ion can be treated independently from others as long as the average distance between the paths is considerably larger than the radii of tracks. Typical doses used in ion-beam therapy are small (Amaldi and Kraft 2005) and the above condition is satisfied (Surdutovich and Solov'yov 2014). Then, the average number of lethal lesions per ion traversing distance $z$ through a cell nucleus is given by a product of $\frac{\mathrm{d} N_{\mathrm{l}}}{\mathrm{d} x}$ and the average length of traverse of all ions passing through a cell nucleus at a given dose,

$$
Y_{1}=\frac{\mathrm{d} N_{\mathrm{l}}}{\mathrm{d} x} \bar{z} N_{\mathrm{ion}}(d) .
$$

The average number of ions traversing the nucleus $N_{\mathrm{ion}}=A_{\mathrm{n}} d / S_{\mathrm{e}}$ depends on dose, LET, and the area of cell nucleus $A_{\mathrm{n}}$.

Combining these expressions, the number of lethal lesions can be written as (Verkhovtsev et al. 2016)

$$
Y_{1}=\frac{\pi}{16} \sigma_{\mathrm{l}}\left(S_{\mathrm{e}}\right) N_{\mathrm{g}} \frac{d}{S_{\mathrm{e}}}
$$

where $N_{\mathrm{g}}$ is genome size, equal to $3.2 \mathrm{Gbp}$ for human cells (Alberts et al. 2014) and to $2.7 \mathrm{Gbp}$ for Chinese hamster cells (Lewis 2013). This expression is obtained by averaging nuclear DNA density over the cell cycle duration. Knowing $N_{\mathrm{g}}$ for a cell line of particular origin and accounting for the chromatin dynamics during the cell cycle, one can evaluate the number density of chromatin $n_{\mathrm{s}}$ [see Verkhovtsev et al. (2016) for details].

The probability of cell survival is given by the probability of zero lethal lesions occurrence, $\Pi_{\text {surv }}=e^{-Y_{1}}$. The logarithm of $\Pi_{\text {surv }}$ with a minus sign is then given by Eq. (12), 


$$
-\ln \Pi_{\text {surv }}=Y_{\mathrm{l}}=\frac{\pi}{16} \sigma_{\mathrm{l}}\left(S_{\mathrm{e}}\right) N_{\mathrm{g}} \frac{d}{S_{\mathrm{e}}} .
$$

This expression relates the empirical parameter $\alpha$ of the LQ model to the physical parameters of the ion projectiles and biological parameters of the target,

$$
\alpha=\frac{\pi}{16} \frac{\sigma_{\mathrm{l}}\left(S_{\mathrm{e}}\right)}{S_{\mathrm{e}}} N_{\mathrm{g}} .
$$

The formalism outlined above describes how to evaluate cell survival probabilities within the MSA in order to compare them with the outcomes of in vitro experiments. Cell survival probabilities and the LET-dependence of $\alpha$, calculated using Eqs. (13), (14), are analyzed below in "Analysis of cell survival curves" and "Dependence of cell survival-related quantities on LET" sections. In our future works we hope to expand this methodology towards the analysis of dose-response curves derived from in vivo experiments.

\section{Consideration of the overkill effect}

It is well known from numerous radiobiological experiments with carbon and heavier ions that RBE does not increase monotonically with LET but has a maximum at $S_{\mathrm{e}} \approx 100-200 \mathrm{keV} / \mu \mathrm{m}$ (depending on the ion type) and gradually decreases at larger $S_{\mathrm{e}}$. This feature is commonly attributed to the so-called "overkill" effect. The explanation of this effect is that at high LET the energy is deposited into a target cell nucleus by a small number of ions, and this energy is larger than that needed for cell inactivation. As a result, such high-LET irradiation produces higher DNA damage than actually required, which leads to a reduction in biological effectiveness (Linz 2012).

Different approaches have been adopted in different radiobiological models to account for this effect. For instance, a "saturation correction" due to non-Poisson distribution of lethal lesions in the cell nucleus was introduced in the LEM and MKM models to describe the radiobiological response to high-LET irradiation (Hawkins 2003; Kase et al. 2008). In the present study, we suggest the following explanation of the overkill effect within the MSA.

To derive the dependence of RBE on LET at large values of stopping power, let us recall the yield of lethal lesions, $Y_{1}$, which is defined by Eqs. (9)-(11). Combining them, one gets

$$
-\ln \Pi_{0}=Y_{1}=n_{\mathrm{s}} \xi S_{\mathrm{e}}^{2} \bar{z} N_{\text {ion }},
$$

where $\Pi_{0}$ is the target cell survival fraction. Notice, that even though $N_{\text {ion }}$ in Eq. (15) is an average number of ions traversing the nucleus, in reality the number of ions is integer. Therefore, $N_{\text {ion }}$ can be redefined as the minimum number of ions required to cause the damage corresponding to $\Pi_{0}$. From Eq. (15) one then derives

$$
N_{\text {ion }}=\left[\frac{-\ln \Pi_{0}}{n_{\mathrm{s}} \xi S_{\mathrm{e}}^{2} \bar{z}}\right]+1,
$$

where square brackets denote the integer part of their content. This expression describes the sufficient minimal number of ions required to produce the target biological effect $\Pi_{0}$. The dose delivered to the cell nucleus by this number of ions is $d=S_{\mathrm{e}} \bar{z} N_{\mathrm{ion}} / m$, where 

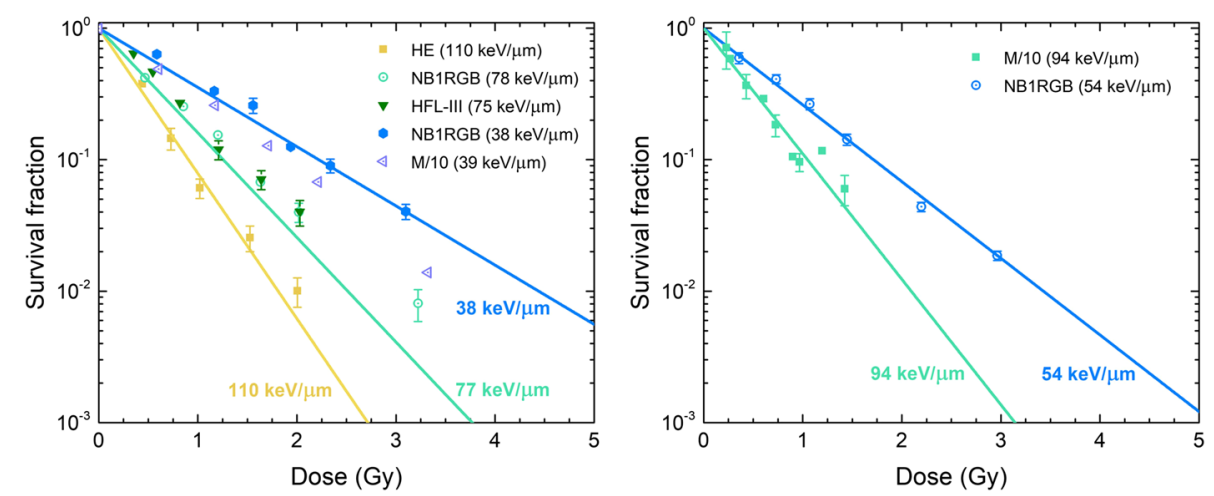

Fig. 1 Survival curves for several normal tissue human cell lines irradiated with monoenergetic carbon ions: human embryonic (HE) fibroblast-like cells, skin fibroblast NB1RGB, normal embryonic lung fibroblast $\mathrm{HFL}-1 \mathrm{II}$, and M/10 cell line derived from the human mammary epithelial H184B cells. The survival probabilities calculated as a function of deposited dose at the indicated values of LET are shown with lines. Experimental data for HE (Suzuki et al. 1996), NB1RGB (Suzuki et al. 2000; Tsuruoka et al. 2005), HFL-III (Suzuki et al. 2000) and M/10 (Belli et al. 2008) cells, measured at a specific dose, are shown by symbols

$\bar{z}$ is the average length of traverse of an ion through the cell nucleus and $m$ is the mass of DNA material therein.

The RBE for a given biological effect, described by a cell survival probability $\Pi_{0}$, can be calculated as follows. According to the LQ model, Eq. (2), the logarithm of a given cell survival probability depends on the photon dose $d_{\gamma}$ of reference radiation as $-\ln \Pi_{0}=\alpha_{\gamma} d_{\gamma}+\beta_{\gamma} d_{\gamma}^{2}$. The RBE is obtained then by dividing $d_{\gamma}$ by the dose due to ions sufficient to achieve $\Pi_{0}$. Using the relations above, this ratio reads as

$$
\mathrm{RBE}=\frac{d_{\gamma}}{S_{\mathrm{e}} \bar{z} N_{\mathrm{ion}} / m}=\frac{d_{\gamma}}{\frac{\mathrm{S}_{\mathrm{e}} \bar{z}}{m}\left(\left[\frac{-\ln \Pi_{0}}{n_{\mathrm{s}} \xi S_{\mathrm{e}}^{2}}\right]+1\right)} .
$$

At small values of LET, $S_{\mathrm{e}} \sim 10^{1} \mathrm{keV} / \mu \mathrm{m}$, the integer part is much larger than unity (i.e., the number of ions incident on a target nucleus $N_{\text {ion }} \gg 1$ ) so that the RBE is a linear function of $S_{\mathrm{e}}$, independent of $\bar{z}$. A linear dependence of RBE on LET corresponds to a linear dependence of the number of lethal lesions on LET, see Eqs. (10) and (12). At larger values of LET, $S_{\mathrm{e}} \sim 10^{2} \mathrm{keV} / \mu \mathrm{m}$ and higher, the integer part gradually approaches zero and RBE becomes asymptotically inversely proportional to $S_{\mathrm{e}}$, i.e., $\mathrm{RBE}=d_{\gamma} m / S_{\mathrm{e}} \bar{z}$. The analysis of RBE at different values of LET is presented in "Evaluation of RBE from cell survival curves "and "Evaluation of RBE at high values of LET" sections.

\section{Results and discussion}

\section{Analysis of cell survival curves}

Figure 1 shows the survival curves for several human normal cell lines irradiated with monoenergetic carbon ions. The survival curves calculated using Eq. (13) are shown with lines. Symbols denote experimental data (Suzuki et al. 1996, 2000; Tsuruoka et al. 2005; Belli et al. 2008) on clonogenic survival of human embryonic (HE) fibroblast-like cells, skin fibroblasts NB1RGB, normal embryonic lung fibroblasts HFL-III, as well as M/10 cells derived from human mammary epithelial cell line H184B. In 
the aforementioned experiments, irradiation was performed in a single fraction at a typical dose rate of $1-2 \mathrm{~Gy} / \mathrm{min}$. The probability of cell inactivation was measured as reproductive cell death using colony-formation assays. After irradiation cells were incubated for the time period from several days up to about two weeks, then fixed and stained. Colonies with more than 50 cells were considered as survivors. Cell survival probability was defined as the ratio between the measured experimental plating efficiency at a given dose to that in non-irradiated control samples.

Figure 1 illustrates that the main trend in the cellular response to ion-beam irradiation at different values of LET is reproduced using the above-described methodology. The results presented in Fig. 1 together with our earlier results (Verkhovtsev et al. 2016) cover a large number of radiobiological experiments compiled in the PIDE database (Friedrich et al. 2013b). This allows us to justify further the applicability of the MSA for the description of macroscopic radiobiological effects of ion-beam irradiation through understanding of the nanoscale mechanisms of ion-induced biodamage.

In this study we have focused on the radiobiological response of normal (i.e., noncancerous) cells. It is assumed that the variation of radiosensitivity (i.e., the variation of survival curves) between the cells of the same origin is rather small and can be neglected in the first approximation. The validity of this assumption is justified below. The variability in radiosensitivity/radioresistance of normal cells of the same origin is much smaller than that of different tumor cell lines (Suzuki et al. 2000). This can be attributed to more frequent mutations in cancerous cells resulting in inactivation of specific repair proteins or underexpression of repair enzymes (Chae et al. 2016). A molecular-level understanding of the mechanisms of DNA damage response to ion irradiation is a complex problem, and we hope that it could be tackled by means of the MSA methodology in our future studies.

Here we consider a number of normal human cells and assume that the density of chromatin and hence the number of complex damage sites do not vary between the different cells. In other words, different normal human cells considered in this work are assumed to have the same amount of DNA material. This allows for the validation of other parameters entering the analytical recipe for the assessment of ion-induced biodamage presented in "The MSA methodology" section. The number density of complex damage sites in the cells is then calculated implying that the nucleus of a typical human normal cell contains $6.4 \times 10^{9}$ base pairs [human diploid cells accommodate two sets of 23 chromosomes, each of which contains about 3.2 Gbp (Alberts et al. 2014)], and this number has been kept constant in the calculations.

It is however expected that the value of $n_{\mathrm{s}}$ may vary significantly between different tumor cell lines of the same origin. This may happen because of an abnormal number of chromosomes (so-called aneuploidy) that is a prominent feature of cancer cells (Thompson and Compton 2011). Due to this effect, chromosomal numbers in tumor cells may differ from healthy tissue cells where the overall karyotype of the cell population remains diploid (Suzuki et al. 2000). As a result, the total amount of DNA material may vary between different cancerous human cells due to aneuploidy and mutations induced. In this case the genome size $N_{\mathrm{g}}$ entering Eqs. (12)-(14) will not be constant but become a variable quantity. A systematic analysis of the genome size 


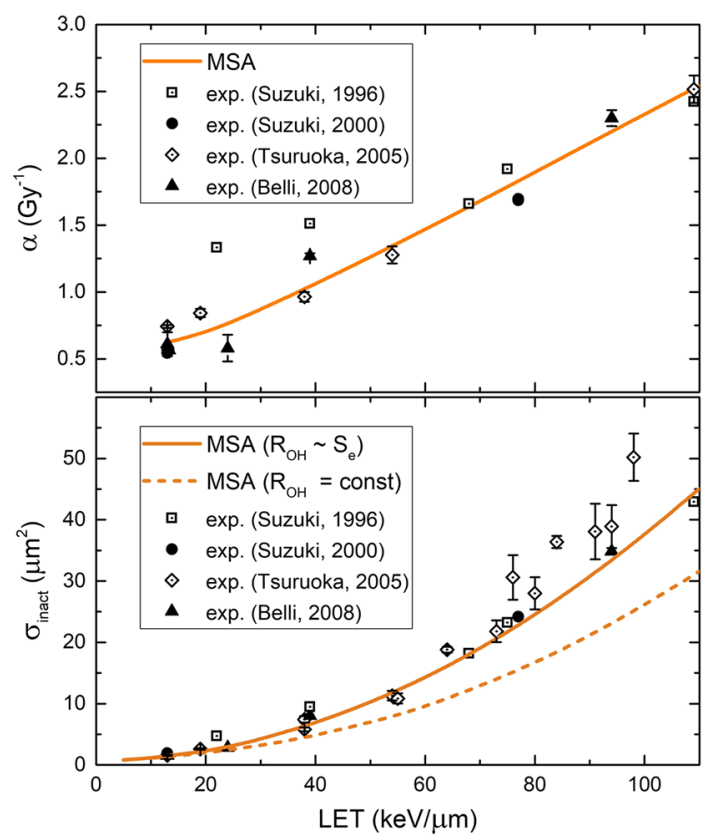

Fig. 2 Slope of the dose-dependent cell survival curve (i.e., the coefficient $\alpha$ in the LQ model) (upper panel) and inactivation cross section $\sigma_{\text {inact }}$ (lower panel) as functions of LET. The MSA results (solid lines) are compared with experimental data from (Suzuki et al. 1996, 2000; Belli et al. 2008; Tsuruoka et al. 2005) (symbols). The dashed line in the lower panel shows the MSA results with a fixed range of reactive species propagation, independent of LET (see the main text for details)

variation in different cancer cells and its impact on the resulting cell survival and RBE will be addressed in our future works.

Figure 1 demonstrates that the assumption made works well for the four cell lines studied. It should be stressed that the MSA-based survival curves were calculated with the same set of parameters described in "The MSA methodology" section, i.e., without adjusting them for each particular experimental dataset with the LQ model. This gives us additional confidence about the robustness of the MSA methodology for the analysis of cell survival.

\section{Dependence of cell survival-related quantities on LET}

The MSA was applied also to analyze other quantities of radiobiological interest. The upper panel of Fig. 2 shows the dependence of the slope of survival curves, $\alpha$, on LET. The results of MSA-based calculations employing Eq. (14) (solid line) are compared with experimental data (Suzuki et al. 1996, 2000; Tsuruoka et al. 2005; Belli et al. 2008) (symbols). Note that the values of $\alpha$ were explicitly given in (Suzuki et al. 2000; Belli et al. 2008) only for a few values of LET. Other experimental figures were extracted from the PIDE database (Friedrich et al. 2013b). The calculated curve agrees well with a data set compiled from the four different experiments. In the lowest-LET limit considered in this work $\left(S_{\mathrm{e}}=13 \mathrm{keV} / \mu \mathrm{m}\right)$ the calculated value $\alpha \approx 0.6$ agrees with the experimental results (Suzuki et al. 2000; Belli et al. 2008). In the LET range up to about $100 \mathrm{keV} / \mu \mathrm{m}$, which is of interest for clinical applications of carbon ions, $\alpha$ gradually increases and reaches 2.5 at $S_{\mathrm{e}}=110 \mathrm{keV} / \mu \mathrm{m}$. As follows from Eq. (14) an increase of $\alpha$ with LET 
reflects a non-linear dependence $\sigma_{\mathrm{l}}\left(S_{\mathrm{e}}\right)$ which is attributed to the indirect mechanism of DNA damage due to the shock wave.

As a by-product of this analysis, we have calculated the inactivation cross section $\sigma_{\text {inact }}$ which is commonly introduced to describe the effects of charged-particle irradiation in terms of ion fluence $F$ instead of dose $d$ (Scholz 2006). The inactivation cross section enters an expression for a fluence-wise definition of cell survival probability, $-\ln \Pi_{\text {surv }}=\sigma_{\text {inact }} F$. Then, using the relation between fluence and dose (Alpen 1997), $d=F S_{\mathrm{e}} / \rho$ (where $\rho$ is the mass density of the target medium), one can calculate the inactivation cross section corresponding to a given level of cell survival as a function of LET,

$$
\sigma_{\text {inact }}=-\frac{\ln \Pi_{\text {surv }} S_{\mathrm{e}}}{d \rho} .
$$

We analyzed $\sigma_{\text {inact }}$ at $37 \%$ survival (this corresponds to an $e$ times decrease of cell survival probability) and compared it with experimental data for the same survival level (Tsuruoka et al. 2005). The survival probabilities at different $S_{\mathrm{e}}$ were calculated using Eq. (13) and the doses corresponding to $\Pi_{\text {surv }}=37 \%$ were obtained from this dependence. These values were used in Eq. (18) to calculate $\sigma_{\text {inact }}$ as a function of LET. This dependence is shown in the lower panel of Fig. 2. Similar to the above-discussed results, the calculated dependence $\sigma_{\text {inact }}\left(S_{\mathrm{e}}\right)$ shows good overall agreement with experimental data. The inactivation cross section depicted by a solid line was calculated assuming that reactive species (free radicals and solvated electrons generated due to interaction of the ion projectile and secondary electrons with water molecules of the medium) are effectively spread away from the ion track via the ion-induced shock wave predicted in Surdutovich and Solov'yov (2010). According to the outcomes of earlier studies (Surdutovich et al. 2017; de Vera et al. 2018), the characteristic range of reactive species propagation increases linearly with LET due to an increasing strength of the shock wave.

The dashed line in the lower panel of Fig. 2 illustrates the cross section $\sigma_{\text {inact }}$ calculated with a fixed range of reactive species propagation, set to $5 \mathrm{~nm}$. This value corresponds to a typical range of diffusion-driven propagation of radical species (mainly $\mathrm{OH}$ radicals) in a cellular environment (Stewart et al. 2011; Nikjoo et al. 1997), which varies in different publications between 4 and $6 \mathrm{~nm}$. The $\mathrm{OH}$ range of $6 \mathrm{~nm}$ was obtained in experiments on X-ray induced DNA strand breaks and cell killing (Roots and Okada 1975), where the average lifetime of $\mathrm{OH}$ radicals was estimated on the order of several nanoseconds. In the LEM IV model an effective range of different radical species is set to a similar value of $4 \mathrm{~nm}$ (Friedrich et al. 2013a). Notice that when the range of reactive species propagation is set to a constant value, the calculated inactivation cross section is systematically smaller than the experimental values.

As discussed above in "Transport of secondary particles" section, shock waves play a significant role in the transport of reactive species due to radial collective flows initiated by them. The analysis of formation and transport of free radicals (Surdutovich and Solov'yov 2015) suggested that in the case of low-LET radiation (where the effects due to the shock waves are expected to be weak) most of the radicals do not leave the ion track since they recombine before they could diffuse out of the track. Reactive molecular dynamics simulations (de Vera et al. 2018) demonstrated that the shock wave-induced 


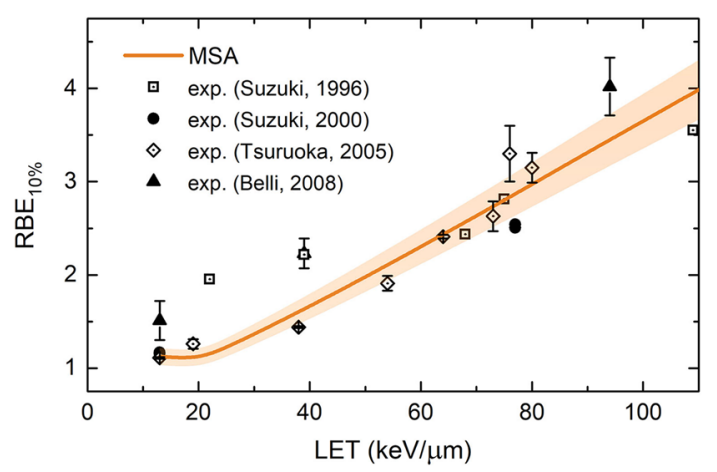

Fig. 3 RBE at 10\% cell survival for human normal tissue cells irradiated with carbon ions. Solid line shows $\mathrm{RBE}_{10 \%}$ calculated by means of the MSA. Shaded area shows a photon dose-related uncertainty due to the difference in the experimental photon response curves (Suzuki et al. 1996, 2000; Tsuruoka et al. 2005; Belli et al. 2008).

collective flow for carbon ions in the Bragg peak region can propagate $\mathrm{OH}$ radicals 80 times faster than diffusion. The outcomes of the present study support further the idea that the shock waves induced by ions traversing a biological medium play a significant role in the indirect mechanisms of ion-induced biodamage on the nanoscale.

Note that the ion-induced shock waves (and the subsequent collective flows) are not considered in existing biophysical models based on the track structure analysis. The latter is based upon the Monte Carlo simulations framework that does not allow to simulate explicitly dynamics of a molecular medium in the vicinity of ion tracks. This makes the direct comparison with track structure based studies rather challenging. However, the results of simulations (de Vera et al. 2018) performed for low-LET protons showed a very good agreement with the Monte Carlo simulations using the Geant4-DNA package, which implements the well-known diffusion-reaction algorithms.

\section{Evaluation of RBE from cell survival curves}

The practical goal of the phenomenon-based assessment of radiation damage by means of the MSA is the calculation of RBE. In this section, the MSA is applied to evaluate RBE for human normal cells irradiated with carbon ions as an illustrative case study. Figure 3 shows the dependence of $\mathrm{RBE}_{10 \%}$, that is RBE corresponding to $10 \%$ cell survival, on LET. $\mathrm{RBE}_{10 \%}$ is one of the most frequently analyzed quantities in radiobiological experiments in vitro. As discussed above, we assume that different normal tissue cells have similar responses to ion-beam radiation. Therefore, for a given value of LET, this response is modeled with a single survival curve. Despite this simplification, this approach gives reasonable results in agreement with experimental data as demonstrated in Figs. 1 and 2. To calculate the RBE, the survival curves obtained by means of the MSA were normalized to the corresponding photon curves taken from each of the four experiments considered (Suzuki et al. 1996, 2000; Tsuruoka et al. 2005; Belli et al. 2008). The photon curves somewhat differ between each other so that the photon dose yielding a $10 \%$ survival varies between 3.4 and 4.0 Gy. This variation leads to a dose-related uncertainty in RBE which is illustrated in Fig. 3 by a shaded area. The solid line shows $\mathrm{RBE}_{10 \%}$ averaged over the four considered experiments. 

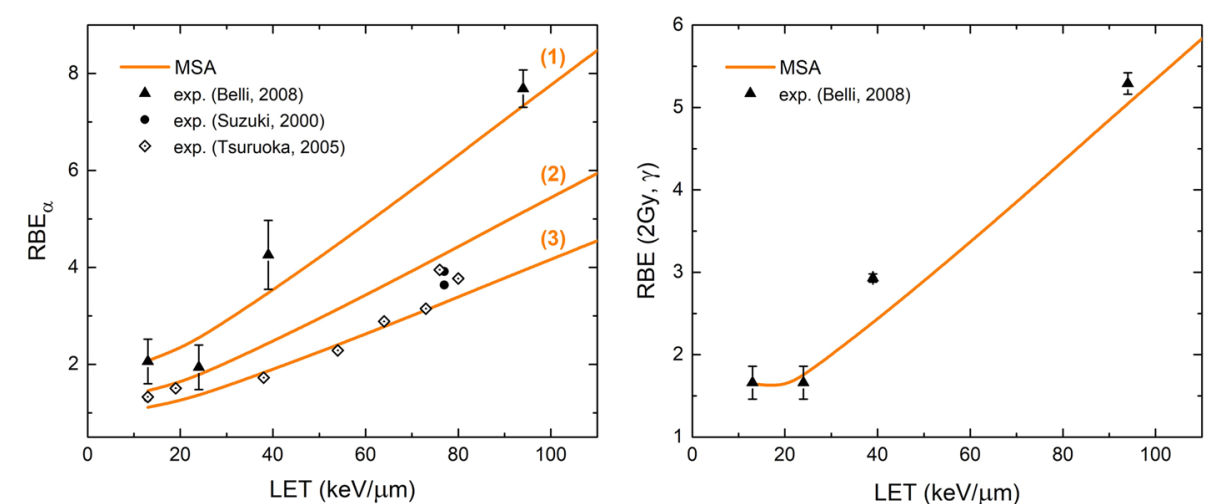

Fig. 4 RBE for human normal cells irradiated with carbon ions: $\operatorname{RBE}_{\alpha}=\alpha_{\text {ion }} / \alpha_{X}$ (left panel) and $\operatorname{RBE}(2 \mathrm{~Gy}, \gamma)$ (right panel). Solid lines show the RBE values calculated by means of the MSA. The RBE $\alpha$ curves denoted as (1), (2), (3) correspond to experimental data (shown by symbols) from Belli et al. (2008), Suzuki et al. (2000) and Tsuruoka et al. (2005), respectively

The experimental values of $\mathrm{RBE}_{10 \%}$ for different normal cells vary from 1.1 to 1.5 in the low-LET region $\left(S_{\mathrm{e}}=13 \mathrm{keV} / \mu \mathrm{m}\right)$ and grow up to 4.0 at $S_{\mathrm{e}} \sim 100 \mathrm{keV} / \mu \mathrm{m}$. The calculated values of RBE follow this trend and are in good overall agrement with experimental results. Note that at low values of LET typical for proton therapy applications $\left(S_{\mathrm{e}} \approx 10-20 \mathrm{keV} / \mu \mathrm{m}\right)$ the calculated $\mathrm{RBE}_{10 \%}$ is almost independent on LET. This can be attributed to the fact that at such low LET the shock wave mechanism is much weaker than in the Bragg peak region of carbon ions, and the transport of radicals (defining the DNA damage) is by and large carried out by diffusion. At higher values of LET the shock wave mechanism starts to play a more important role that leads to an increased number of lethal lesions and thus the RBE. The calculated RBE matches the experimental figures accounting for the experimental error bars and the dose-related uncertainty due to averaging over the four different photon curves.

It is also common to consider other quantities of radiobiological interest, such as RBE at different levels of cell inactivation (e.g., $50 \%, 37 \%$, or $1 \%$ ), $\mathrm{RBE}_{\alpha}=\alpha_{\text {ion }} / \alpha_{\mathrm{X}}$ (which describes the ion biological effectiveness at low doses), and $\operatorname{RBE}(2 \mathrm{~Gy}, \gamma)$ that is the RBE at a given ion dose leading to the same inactivation level as produced by the photon dose of 2 Gy (Kase et al. 2008; Belli et al. 2000). The latter is regarded as a more relevant quantity for clinical applications because $\operatorname{RBE}(2 \mathrm{~Gy}, \gamma)$ corresponds to the typical dose used in fractionated-dose protocols. The evaluation of different RBE specifications provides a playground to test further the accuracy of the MSA methodology. Figure 4 shows the $\operatorname{RBE}_{\alpha}$ (left panel) and $\operatorname{RBE}(2 \mathrm{~Gy}, \gamma)$ (right panel) for carbon ions as functions of LET. The calculated curves are compared to the experimental data for NB1RGB (Suzuki et al. 2000; Tsuruoka et al. 2005) and M/10 cells (Belli et al. 2008). Note that the latter is the only reference out of the three experiments considered where the data on $\operatorname{RBE}_{\alpha}$ and $\operatorname{RBE}(2 \mathrm{~Gy}, \gamma)$ has been given explicitly. To compare with other experimental results, the corresponding values of $\alpha_{\mathrm{X}}$ were taken from the PIDE database. The experimental values of $\mathrm{RBE}_{\alpha}$ vary between 1.1 and 2.0 for low-LET carbon-ion radiation and increase by the factor of four in the Bragg peak region. The $\operatorname{RBE}(2 \mathrm{~Gy}, \gamma)$ has a similar trend and increases from 1.6 up to about 5.5. The calculated dependencies of $\operatorname{RBE}_{\alpha}$ and $\operatorname{RBE}(2 \mathrm{~Gy}, \gamma)$ on LET are in overall 

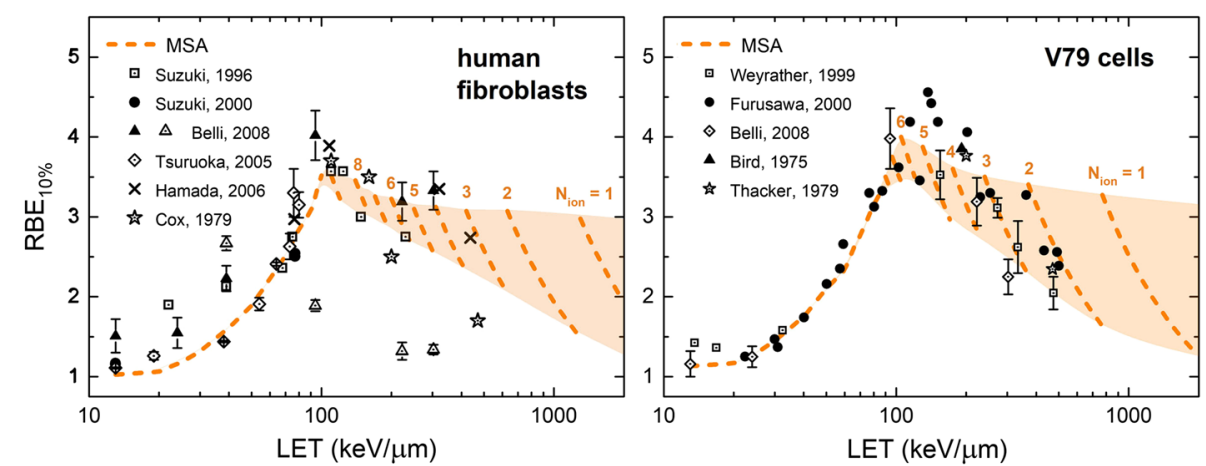

Fig. 5 RBE at 10\% cell survival for human normal tissue cells (left panel) and Chinese hamster V79 cells (right panel) irradiated with carbon ions. The results are obtained by means of Eq. (17). In the high-LET region the RBE becomes inversely proportional to LET, and the absolute values of RBE depend on the number of ions that traverse a cell nucleus. The values of $N_{\text {ion }}$ corresponding to different segments of the calculated curve are indicated. Symbols depict experimental data from Suzuki et al. $(1996,2000)$, Tsuruoka et al. (2005), Belli et al. (2008), Cox and Masson (1979) and Hamada et al. (2006) (for human fibroblasts) and from Weyrather et al. (1999), Furusawa et al. (2000), Bird and Burki (1975) and Thacker et al. (1979) (for V79). The shaded area shows the variation of RBE with an account for statistical uncertainties in the number of ions traversing a cell nucleus and the LET (see the text for details)

agreement with the results presented in Suzuki et al. (2000), Tsuruoka et al. (2005) and Belli et al. (2008).

\section{Evaluation of RBE at high values of LET}

In this section we analyze the RBE in the overkill regime. The left panel of Fig. 5 shows $\mathrm{RBE}_{10 \%}$ for human fibroblasts in a broad LET range up to $2000 \mathrm{keV} / \mu \mathrm{m}$. The curves calculated by means of Eq. (17) are compared with experimental data described in Fig. 3 and those extended towards the larger values of LET as well as with other experimental data from Cox and Masson (1979) and Hamada et al. (2006). At small and moderate values of LET (up to about $80 \mathrm{keV} / \mu \mathrm{m}$ ) the dependence of RBE on LET corresponds to the results shown in Fig. 3 (note the semi-logarithmic plot in Fig. 5 which transforms the linear dependence shown in Fig. 3 into a power law function). At larger $S_{\mathrm{e}}$ the RBE starts to deviate from the linear dependence as the number of ions traversing the nucleus becomes comparable (in the order of magnitude) to 1 . With an increase of LET a small number of ions is needed to deliver the dose that would inactivate the cell. Since the number of ions hitting the nucleus is an integer quantity, the $\operatorname{RBE}\left(S_{\mathrm{e}}\right)$ dependence transforms into a series of segments of hyperbolas, where each segment corresponds to a given number $N_{\text {ion }}$. The values of $N_{\text {ion }}$ corresponding to different hyperbolas are indicated in the figure.

The dependence presented shows good overall agreement with experimental data for different human fibroblasts-HE, NB1RGB, HFL-III, M/10, and AG01522 (Suzuki et al. 1996; Belli et al. 2008; Cox and Masson 1979; Hamada et al. 2006). An experimental dataset for the HF-19 cell line from Belli et al. (2008) (open triangles) has a very different behavior, namely the maximum value of RBE of 2.7 at $S_{\mathrm{e}} \approx 40 \mathrm{keV} / \mu \mathrm{m}$ and a rapid decrease down to 1.3 at $S_{\mathrm{e}} \approx 200 \mathrm{keV} / \mu \mathrm{m}$. This dependence deviates 
strongly from the other experimental data including the results from Cox and Masson (1979) (open stars) for the same HF-19 cell line. Apart from this deviation, the MSA results are in agreement with all other experimental numbers.

We also analyzed the overkill effect in the Chinese hamster V79 cells, see the right panel of Fig. 5. The calculated dependence $\operatorname{RBE}\left(S_{\mathrm{e}}\right)$ was compared with experimental data (Weyrather et al. 1999; Furusawa et al. 2000; Bird and Burki 1975; Thacker et al. 1979) extracted from the PIDE database. The experimental data for V79 cells are less scattered compared to the human fibroblasts described above. The MSA captures the main trend of the $\operatorname{RBE}\left(S_{\mathrm{e}}\right)$ dependence, namely the maximum at $S_{\mathrm{e}} \approx 100-150 \mathrm{keV} / \mu \mathrm{m}$ followed by a gradual decrease at higher LET. Note that at intermediate LET values, the MSA calculations for the V79 cells correlate also with the calculations performed using the LEM and MKM models. In Kase et al. (2008) the RBE $10 \%$ was calculated for the case of proton and helium ion irradiation in the LET range up to about $150 \mathrm{keV} / \mu \mathrm{m}$. At this value, the RBE for $\alpha$-particles predicted by the MKM was about 4.7 while the LEM predicted a smaller value of about 3.7. These numbers are very close to those predicted by the present MSA calculations.

An important observation is that in the high-LET regime the dose needed to achieve a given biological effect is deposited by only a few ions, and the number of ions $N_{\text {ion }}$ is a discrete quantity. The fact that a given number of ions may produce more damage than would be needed for a given effect leads to a significant (up to 20\%) variation in RBE. As follows from Fig. 5 the variation of RBE has been commonly observed in experiments. This effect may be taken into consideration in the analysis of experimental data on smalldose irradiation with high-LET ions.

In in vitro experiments with pencil-beam radiation, $N_{\text {ion }}$ and $S_{\mathrm{e}}$ are stochastic quantities that vary randomly within the beam. To account for this, the above-described analysis can be extended by calculating the variation of RBE (17) due to statistical uncertainties of $N_{\text {ion }}$ and $S_{\mathrm{e}}$. Typical pencil beams used in proton or carbon-ion therapy have the lateral size of several millimeters (Hoppe et al. 2010). Taking as an estimate a typical cell diameter of about $20-50 \mu \mathrm{m}$, one gets that $N_{\text {cell }} \sim 10^{3}-10^{4}$ cells will be irradiated by such a beam. For $N_{\text {cell }} \gg 1$ the number of ions hitting a cell can be evaluated as $N_{\text {ion }}=\bar{N}_{\text {ion }} \pm \Delta N_{\text {ion }} \approx \bar{N}_{\text {ion }} \pm \sqrt{\bar{N}_{\text {ion }} / N_{\text {cell }}}$, where $\bar{N}_{\text {ion }}$ is defined by Eq. (16). For $N_{\text {cell }} \sim 10^{3}$, the variation $\Delta N_{\text {ion }}$ does not exceed $1.5 \%$ which leads to a minor variation of RBE compared to the data presented in Fig. 5. The variation of LET can be extracted from experimental data, e.g., the value of $77 \pm 1.8 \mathrm{keV} / \mu \mathrm{m}\left(\Delta S_{\mathrm{e}}=2.3 \%\right)$ was reported in Suzuki et al. (2000). It is expected that the statistical error for LET will grow with an increase of LET. However, the publications on irradiation of cells with high-LET carbon ions, which are used for the comparison in this study, provided only the averaged values of LET but not the uncertainties. To estimate a magnitude of this variation at high LET, we used the numbers provided in Dang et al. (2011) for irradiation of plasmid DNA with carbon ions at the spread-out Bragg peak $\left(S_{\mathrm{e}} \approx 189 \mathrm{keV} / \mu \mathrm{m}\right)$. In that paper the experimental uncertainty of LET was about $8 \%$. The shaded areas in Fig. 5 show the $\mathrm{RBE}_{10 \%}$ for human fibroblasts and V79 cells with the statistical uncertainty in LET taken into account. Based on the available experimental data on $\Delta S_{\mathrm{e}}$, the uncertainty was set to $2.5 \%$ at the LET of about $100 \mathrm{keV} / \mu \mathrm{m}$ and increased gradually up to $8 \%$ at higher LET values. The uncertainty in LET leads to a broadening of the segments of hyperbolas 
shown in Fig. 5, especially in the region of high LET where $\Delta S_{\mathrm{e}}$ is large. As a result, the RBE as a function of LET transforms into a quasi-continuous band depicted by the shaded area.

\section{Conclusions}

In this study the multiscale approach to the physics of radiation damage with ions was applied to calculate relative biological effectiveness for normal human and Chinese hamster cells irradiated with carbon ions at different values of LET. As a by-product of this analysis, other radiobiological parameters such as inactivation cross section were calculated and compared with available experimental data. Normal cell lines have been chosen as a case study because their proliferation is highly organized as compared to tumor cells. This allowed us to test robustness of the MSA-based methodology and validate its key parameters, e.g., the genome size which remains almost constant in different normal cells of the same origin but may vary greatly in different tumor cells.

We also tested the hypothesis that the response of different normal cells to ionbeam irradiation does not vary significantly and thus can be described by a single survival curve for each value of LET. Good agreement with a large set of experimental data on clonogenic cell survival, inactivation cross section, and different RBE specifications $\left(\mathrm{RBE}_{10 \%}, \mathrm{RBE}_{\alpha}\right.$ and $\left.\mathrm{RBE}(2 \mathrm{~Gy}, \gamma)\right)$, taken from the Particle Irradiation Data Ensemble database, allows us to conclude about the predictive power of the MSA.

Finally, the MSA was utilized to describe the "overkill" effect which results in a decrease of RBE at high values of LET. The results obtained for different human fibroblasts and Chinese hamster V79 cells are in agreement with experimental data. We demonstrated that for a given number of high-LET ions traversing a cell nucleus the RBE becomes inversely proportional to LET. The fact that a given number of ions may produce more damage than would be needed for a given biological effect leads to a significant (up to $20 \%$ ) variation in RBE. This effect can be considered in the analysis of experimental data on small-dose irradiation with high-LET ions as it may lead to re-evaluation of the RBE in the high-LET regime.

Abbreviations

DSB: double-strand break; IBCT: ion-beam cancer therapy; LET: linear energy transfer; LEM: local effect model; MKM: microdosimetric kinetic model; MMKM: modified MKM; MSA: the multiscale approach to the physics of radiation damage with ions; PIDE: particle irradiation data ensemble database; RBE: relative biological effectiveness; SSB: single-strand break.

Acknowledgements

AV acknowledges the DKFZ International Postdoc Program.

Authors' contributions

AV and ES performed the analysis and drafted the manuscript. AVS supervised the work. All authors contributed to the conceptual design of the work, discussion of the results and preparation of the manuscript. All authors read and approved the final manuscript.

Funding

This work was supported through the DKFZ Postdoctoral Fellowship (granted to AV) and by the Deutsche Forschungsgemeinschaft.

Availability of data and materials

Not applicable.

Ethics approval and consent to participate

Not applicable. 


\section{Consent for publication}

Not applicable.

\section{Competing interests}

The authors declare that they have no competing interests.

\section{Author details}

${ }^{1}$ Division of Medical Physics in Radiation Oncology, German Cancer Research Center (DKFZ), Im Neuenheimer Feld 280, 69120 Heidelberg, Germany. ${ }^{2}$ MBN Research Center, Frankfurt Innovation Center for Biotechnology, Altenhöferallee 3, 60438 Frankfurt am Main, Germany. ${ }^{3}$ Ioffe Institute, Politekhnicheskaya 26, 194021 St. Petersburg, Russia. ${ }^{4}$ Department of Physics, Oakland University, 2200 N. Squirrel Rd., Rochester, MI 48309, USA.

Received: 6 February 2019 Accepted: 8 May 2019

Published online: 23 May 2019

\section{References}

Alberts B, Johnson A, Lewis J, Morgan D, Raff M, Roberts K, Walter P. Molecular biology of the cell. 6th ed. New York: Garland Science; 2014.

Alpen E. Radiation biophysics. 2nd ed. San Diego: Academic Press; 1997.

Amaldi U, Kraft G. Radiotherapy with beams of carbon ions. Rep Prog Phys. 2005;68:1861-82.

Belli M, Bettega D, Calzolari P, et al. Inactivation of human normal and tumour cells irradiated with low energy protons. Int J Radiat Biol. 2000;76:831-9.

Belli M, Bettega D, Calzolari P, et al. Effectiveness of monoenergetic and spread-out Bragg peak carbon-ions for inactivation of various normal and tumour human cell lines. J Radiat Res. 2008;49:597-607.

Bird RP, Burki HJ. Survival of synchronized Chinese hamster cells exposed to radiation of different linear-energy transfer. Int J Radiat Biol Relat Stud Phys Chem Med. 1975;27:105-20.

Bodgi L, Canet A, Pujo-Menjouet L, Lesne A, Victor J-M, Foray N. Mathematical models of radiation action on living cells: from the target theory to the modern approaches. A historical and critical review. J Theor Biol. 2016;394:93-101.

Brahme A, Lind BK. A systems biology approach to radiation therapy optimization. Radiat Environ Biophys. 2010:49:111-24.

Carlson DJ, Stewart RD, Semenenko VA, Sandison GA. Combined use of Monte Carlo DNA damage simulations and deterministic repair models to examine putative mechanisms of cell killing. Radiat Res. 2008;169:447-59.

Chae YK, Anker JF, Carneiro BA, Chandra S, Kaplan J, Kalyan A, Santa-Maria CA, Platanias LC, Giles FJ. Genomic landscape of DNA repair genes in cancer. Oncotarget. 2016;7:23312-21.

Cox R, Masson WK. Mutation and inactivation of cultured mammalian cells exposed to beams of accelerated heavy ions. III. Human diploid fibroblasts. Int J Radiat Biol Relat Stud Phys Chem Med. 1979;36:149-60.

Cucinotta FA, Nikjoo H, Goodhead DT. Applications of amorphous track models in radiation biology. Int J Radiat Biol. 1999;38:81-92.

Curtis SB. Lethal and potentially lethal lesions induced by radiation—a unified repair model. Radiat Res. 1986;106:252-70.

Dang HM, van Goethem MJ, van der Graaf ER, Brandenburg S, Hoekstra R, Schlathölter T. Heavy ion induced damage to plasmid DNA: plateau region vs. spread out Bragg-peak. Eur Phys J D. 2011;63:359-67.

de Vera P, Mason NJ, Currell FJ, Solov'yov AV. Molecular dynamics study of accelerated ion-induced shock waves in biological media. Eur Phys J D. 2016;70:183.

de Vera P, Surdutovich E, Mason NJ, Currell FJ, Solov'yov AV. Simulation of the ion-induced shock waves effects on the transport of chemically reactive species in ion tracks. Eur Phys J D. 2018;72:147.

de Vera P, Surdutovich E, Solov'yov AV. The role of shock waves on the biodamage induced by ion beam radiation. 2019. (Submitted to the topical series "Multiscale Modelling for Cancer Radiotherapies: from X-rays to ions" of Cancer Nanotechnology).

Elsässer T, Krämer M, Scholz M. Accuracy of the local effect model for the prediction of biologic effects of carbon ion beams in vitro and in vivo. Int J Radiat Oncol Biol Phys. 2008;71:866-72.

Fraile A, Smyth M, Kohanoff J, Solov'yov AV. First principles simulation of damage to solvated nucleotides due to shock waves. J Chem Phys. 2019;150:015101.

Frese MC, Yu VK, Stewart RD, Carlson DJ. A mechanism-based approach to predict the relative biological effectiveness of protons and carbon ions in radiation therapy. Int J Radiat Oncol Biol Phys. 2012;83:442-50.

Friedland W, Kundrát P, Jacob P. Stochastic modelling of DSB repair after photon and ion irradiation. Int J Radiat Biol. 2012;88:129-36.

Friedland W, Schmitt E, Kundrát P, Dingfelder M, Baiocco G, Barbieri S, Ottolenghi A. Comprehensive track-structure based evaluation of DNA damage by light ions from radiotherapy-relevant energies down to stopping. Sci Rep. 2017;7:45161.

Friedrich T, Scholz U, Elsässer T, Durante M, Scholz M. Calculation of the biological effects of ion beams based on the microscopic spatial damage distribution pattern. Int J Radiat Biol. 2012;88:103-7.

Friedrich T, Grün R, Scholz U, Elsässer T, Durante M, Scholz M. Sensitivity analysis of the relative biological effectiveness predicted by the local effect model. Phys Med Biol. 2013a;58:6827-49.

Friedrich T, Scholz U, Elsässer T, Durante M, Scholz M. Systematic analysis of RBE and related quantities using a database of cell survival experiments with ion beam irradiation. J Radiat Res. 2013b;54:494-504.

Furusawa Y, Fukutsu K, Aoki M, Itsukaichi H, Eguchi-Kasai K, Ohara H, Yatagai F, Kanai T, Ando K. Inactivation of aerobic and hypoxic cells from three different cell lines by accelerated ${ }^{3} \mathrm{He}-,{ }^{12} \mathrm{C}$ - and ${ }^{20} \mathrm{Ne}-i o n$ beams. Radiat Res. 2000;154:485-96.

Hall EJ, Giaccia AJ. Radiobiology for the radiologist. 8th ed. Philadelphia: Wolters Kluwer; 2018. 
Hamada N, Funayama T, Wada S, Sakashita T, Kakizaki T, Ni M, Kobayashi Y. LET-dependent survival of irradiated normal human fibroblasts and their descendents. Radiat Res. 2006;166:24-30.

Hawkins RB. A microdosimetric-kinetic model of cell death from exposure to ionizing radiation of any LET, with experimental and clinical applications. Int J Radiat Biol. 1996;69:739-55.

Hawkins RB. A microdosimetric-kinetic model for the effect of non-Poisson distribution of lethal lesions on the variation of RBE with LET. Radiat Res. 2003;160:61-9.

Hoppe RT, Phillips TL, Roach M, editors. Leibel and Phillips textbook of radiation oncology. 3rd ed. Philadelphia: Elsevier Saunders; 2010.

Huels MA, Boudaïffa B, Cloutier P, Hunting D, Sanche L. Single, double, and multiple double strand breaks induced in DNA by 3-100 eV electrons. J Am Chem Soc. 2003;125:4467-77.

IAEA Technical Reports Series 461. Relative biological effectiveness in ion beam therapy. Vienna: International Atomic Energy Agency. 2008.

Inaniwa T, Furukawa T, Kase Y, Matsufuji N, Toshito T, Matsumoto Y, Furusawa Y, Noda K. Treatment planning for a scanned carbon beam with a modified microdosimetric kinetic model. Phys Med Biol. 2010;55:6721-37.

International Commission of Radiation Units and Measurements. ICRU Report 16 "Linear Energy Transfer". Washington, D.C.; 1970.

Jensen AD, Münter MW, Debus J. Review of clinical experience with ion beam radiotherapy. Br J Radiol. 2011;84:S035-47. Jäkel O, Karger CP, Debus J. The future of heavy ion radiotherapy. Med Phys. 2008;35:5653-63.

Karger CP, Peschke P. RBE and related modeling in carbon-ion therapy. Phys Med Biol. 2018;63:01TR02.

Kase Y, Kanai T, Matsufuji N, Furusawa Y, Elsässer T, Scholz M. Biophysical calculation of cell survival probabilities using amorphous track structure models for heavy-ion irradiation. Phys Med Biol. 2008;53:37-59.

Kase Y, Kanai T, Sakama M, Tameshige Y, Himukai T, Nose H, Matsufuji N. Microdosimetric approach to NIRS-defined biological dose measurement for carbon-ion treatment beam. J Radiat Res. 2011;52:59-68.

Kelleler AM. Fundamentals of microdosimetry. In: Kase KR, Bjärngard BE, Attix FH, editors. The Dosimetry of lonizing Radiation, vol. 1. San Diego: Academic Press; 1985. p. 77-162.

Kops GJPL, Weaver BAA, Cleveland DW. On the road to cancer: aneuploidy and the mitotic checkpoint. Nat Rev Cancer. 2005;5:773-85.

Lewis NE, et al. Genomic landscapes of Chinese hamster ovary cell lines as revealed by the Cricetulus griseus draft genome. Nat Biotechnol. 2013;31:759-65.

Lind BK, Persson LM, Edgren MR, Hedlöf I, Brahme A. Repairable-conditionally repairable damage model based on dual Poisson processes. Radiat Res. 2003;160:366-75.

Linz U, editor. Ion beam therapy: fundamentals, technology. In: Clinical applications. Berlin: Springer; 2012.

Loeffler JS, Durante M. Charged particle therapy_optimization, challenges and future directions. Nat Rev Clin Oncol. 2013;10:411-24.

Malyarchuk S, Castore R, Harrison L. Apex1 can cleave complex clustered DNA lesions in cells. DNA Repair. 2009;8:1343-54.

Mohamad O, Sishc BJ, Saha J, et al. Carbon ion radiotherapy: a review of clinical experiences and preclinical research, with an emphasis on DNA damage/repair. Cancers. 2017;9:66.

Nikjoo H, O'Neill P, Goodhead DT, Terrissol M. Computational modelling of low-energy electron-induced DNA damage by early physical and chemical events. Int J Radiat Biol. 1997;71:467-83.

Paganetti H. Relative biological effectiveness (RBE) values for proton beam therapy. Variations as a function of biological endpoint, dose, and linear energy transfer. Phys Med Biol. 2014;59:R419-72.

Particle Therapy Co-Operative Group (PTCOG). Particle therapy facilities in clinical operation. https://www.ptcog.ch/index php/facilities-in-operation. Accessed 04/2019.

Particle Therapy Co-Operative Group (PTCOG). Particle therapy facilities under construction. https://www.ptcog.ch/index .php/facilities-under-construction. Accessed 04/2019.

Roots R, Okada S. Estimation of life times and diffusion distances of radicals involved in X-ray-induced DNA strand breaks or killing of mammalian cells. Radiat Res. 1975;64:306-20.

Saager M, Peschke P, Welzel T, Huang L, Brons S, Grün R, Scholz M, Debus J, Karger CP. Late normal tissue response in the rat spinal cord after carbon ion irradiation. Radiat Oncol. 2018;13:5.

Sage E, Harrison L. Clustered DNA lesion repair in eukaryotes: relevance to mutagenesis and cell survival. Mutat Res. 2011;711:123-33.

Sanche L. Low energy electron-driven damage in biomolecules. Eur Phys J D. 2005;35:367-90.

Schardt D, Elsässer T, Schulz-Ertner D. Heavy-ion tumor therapy: physical and radiobiological benefits. Rev Mod Phys. 2010:82:383-425.

Scholz M. Dose response of biological systems to low- and high-LET radiation. In: Horowitz Y, editor. Microdosimetric response of physical and biological systems to low- and high-LET radiations: theory and applications to dosimetry. Amsterdam: Elsevier; 2006. p. 1-73.

Scholz M, Kellerer AM, Kraft-Weyrater W, Kraft G. Computation of cell survival in heavy ion beams for therapy. Radiat Environ Biophys. 1997;36:59-66.

Solov'yov AV, editor. Nanoscale insights into ion-beam cancer therapy. Cham: Springer International Publishing; 2017.

Solov'yov AV, Surdutovich E, Scifoni E, Mishustin I, Greiner W. Physics of ion beam cancer therapy: a multiscale approach. Phys Rev E. 2009;79:011909.

Stewart RD, Yu VK, Georgakilas AG, Koumenis C, Park JH, Carlson DJ. Effects of radiation quality and oxygen on clustered DNA lesions and cell death. Radiat Res. 2011;176:587-602.

Surdutovich E, Solov'yov AV. Shock wave initiated by an ion passing through liquid water. Phys Rev E. 2010;82:051915.

Surdutovich E, Solov'yov AV. Double strand breaks in DNA resulting from double ionization events. Eur Phys J D. 2012;66:206.

Surdutovich E, Solov'yov AV. Multiscale approach to the physics of radiation damage with ions. Eur Phys J D. 2014;68:353.

Surdutovich E, Solov'yov AV. Transport of secondary electrons and reactive species in ion tracks. Eur Phys J D. 2015;69:193. 
Surdutovich E, Solov'yov AV. Cell survival probability in a spread-out Bragg peak for novel treatment planning. Eur Phys J D. 2017;71:210.

Surdutovich E, Gallagher DC, Solov'yov AV. Calculation of complex DNA damage induced by ions. Phys Rev E. 2011;84:051918.

Surdutovich E, Yakubovich AV, Solov'yov AV. Biodamage via shock waves initiated by irradiation with ions. Sci Rep. 2013;3:1289.

Surdutovich E, Verkhovtsev A, Solov'yov AV. Ion-impact-induced multifragmentation of liquid droplets. Eur Phys J D. 2017;71:285.

Sushko GB, Solov'yov IA, Verkhovtsev AV, Volkov SN, Solov'yov AV. Studying chemical reactions in biological systems with MBN Explorer: implementation of molecular mechanics with dynamical topology. Eur Phys J D. 2016;70:12.

Suzuki M, Watanabe M, Kanai T, Kase Y, Yatagai F, Kato T, Matsubara S. LET dependence of cell death, mutation induction and chromatin damage in human cells irradiated with accelerated carbon ions. Adv Space Res. 1996;1 18:127-36.

Suzuki M, Kase Y, Yamaguchi H, Kanai T, Ando K. Relative biological effectiveness for cell-killing effect on various human cell lines irradiated with heavy-ion medical accelerator in Chiba (HIMAC) carbon-ion beams. Int J Radiat Oncol Biol Phys. 2000;48:241-50.

Thacker J, Stretch A, Stephens MA. Mutation and inactivation of cultured mammalian cells exposed to beams of accelerated heavy ions. II. Chinese hamster V79 cells. Int J Radiat Biol Relat Stud Phys Chem Med. 1979;36:137-48.

Thompson SL, Compton DA. Chromosomes and cancer cells. Chromosom Res. 2011;19:433-44.

Tsuruoka C, Suzuki M, Kanai T, Fujitaka K. LET and ion species dependence for cell killing in normal human skin fibroblasts. Radiat Res. 2005;163:494-500.

Verkhovtsev A, Surdutovich E, Solov'yov AV. Multiscale approach predictions for biological outcomes in ion-beam cancer therapy. Sci Rep. 2016:6:27654

von Sonntag C. The chemical basis of radiation biology. London: Taylor \& Francis; 1987.

Ward JF. Radiation mutagenesis: the initial DNA lesions responsible. Radiat Res. 1995;142:362-8.

Weyrather WK, Ritter S, Scholz M, Kraft G. RBE for carbon track-segment irradiation in cell lines of differing repair capacity. Int J Radiat Biol. 1999;75:1357-64.

\section{Publisher's Note}

Springer Nature remains neutral with regard to jurisdictional claims in published maps and institutional affiliations.

- fast, convenient online submission

- thorough peer review by experienced researchers in your field

- rapid publication on acceptance

- support for research data, including large and complex data types

- gold Open Access which fosters wider collaboration and increased citations

- maximum visibility for your research: over 100M website views per year

At BMC, research is always in progress.

Learn more biomedcentral.com/submissions 